MODELING, IDENTIFICATION AND CONTROL, 1992, vOL. 13, NO. 2, 65-76

doi:10.4173/mic.199221

\title{
Multiphase flow metering using capacitance transducer and multivariate calibration
}

\author{
ØYVIND MIDTTVEIT $\dagger$, VIKTOR BERGE $\ddagger$ \\ and EIVIND DYKESTEEN $\dagger$
}

Keywords: Process measurements, signal processing, feature extraction, chemometrics, multi-level modeling.

\begin{abstract}
The method of multivariate calibration is experimentally investigated to establish estimators of the required pertinent flow parameters in multiphase pipe flow. The unfiltered primary signals, provided by a capacitance sensor, are analysed as discrete time series and the signal characteristics are extracted. The multivariate model that is generated estimates the flow composition based on the extracted information existing in the broad-band capacitance signal. The data analysis and test results are presented.
\end{abstract}

\section{Introduction}

In the oil production industry multiphase flow metering is generally required both for reservoir management purposes and for allocation between partners. During the last years Chr. Michelsen Institute (CMI) has developed non-intrusive capacitance sensors for accurate measurements of water fractions in two phase oil/water mixtures. A parallel project during the period 1985-1990 resulted in an industrial prototype multiphase fraction meter capable of measuring the composition of a well mixed oil/gas/water flow.

The CMI multiphase fraction meter consists of a capacitance sensor to measure the mean permittivity (dielectric constant) of the mixture and a gamma densitometer to measure the mean density. From these two independent measurements, and knowing that the sum of all the fractions will always be equal to one, the individual fractions of oil, gas and water can be determined (Dykesteen and Frantzen 1990).

When measuring the mean permittivity, only the mean value, i.e. the low-pass filtered version of the capacitance signals, is utilized, thus some information that is latent in the capacitance signals is then lost due to averaging. The basic idea of the alternative approach presented here, is that the utilization of information in the unfiltered capacitance time series signals may make it possible to measure the flow composition using the capacitance sensor only, thereby excluding the need for a gamma densitometer.

Multiphase flow is undoubtedly a very complex flow phenomenon (Taitel et al. 1980). The corresponding features of the capacitance signals reflect this complexity and represent a pattern containing information on the behaviour of the multiphase flow. The capacitance signal under different flow conditions is schematically illustrated in

Received 12 December 1991.

† Chr. Michelsen Institute (CMI), Fantoftvegen 38, N-5036 Fantoft, Norway.

$\ddagger$ Formerly CMI, now Division of Engineering Cybernetics, The Norwegian Institute of Technology, N-7034 Trondheim, Norway. 


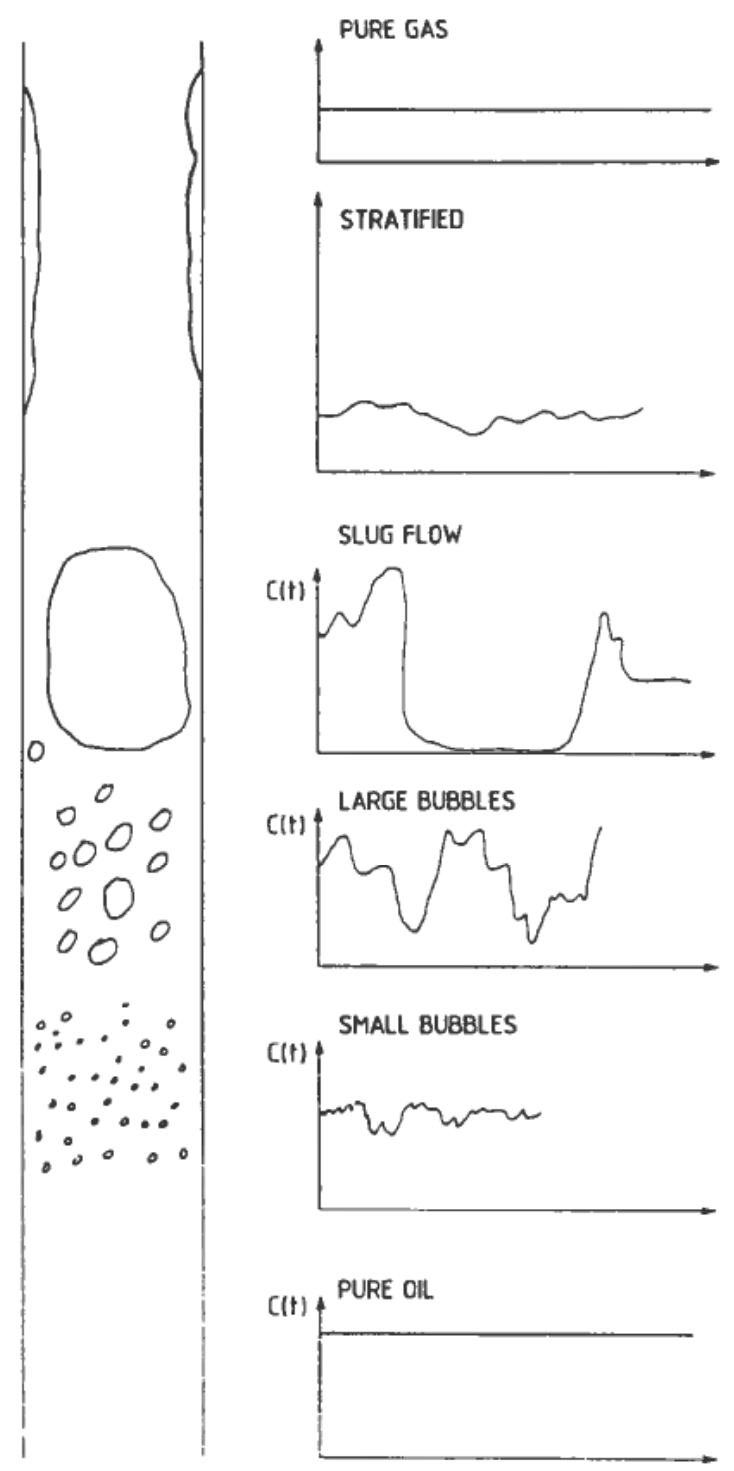

Figure 1. The capacitance signal under different flow conditions.

Fig. 1. In a three-component flow where gas is present, a rise in the variance of the capacitance signals has been observed with increasing gas content (Hammer 1983), and capacitance changes are also generally found with variations in flow regimes (and flow rates) as shown in principle in Fig. 1. Hence, the variance of the capacitance signal is likely to be mostly correlated to the gas content of the multiphase flow. The permittivities of oil and gas are normally $2 \cdot 2-2 \cdot 5$ and 1, respectively, while the permittivity for process water varies from 60 to 81 depending on the salinity. A consequence of this is that the dc-level of the capacitance signal is mostly susceptible to the water fraction. Hence, multiphase flow is a highly coupled process where compositions, flow rates and flow patterns all mutually interact.

This paper will show that multivariate calibration may offer a signal decoupling routine capable of identifying the gas and water fractions, with an acceptable uncertainty, that is only based on the signals provided by a single capacitance sensor. 


\section{Multivariate calibration}

In Multivariate Calibration (MC) mathematical relations between two data sets are found based on statistical modeling. Since the relations to a large degree are based on statistics, the techniques are frequently called soft modeling as opposed to hard modeling which reflects applications where physical laws mainly are allowed to describe the process. Different regression techniques may be applied as Multiple Linear Regression (MLR), Principal Component Regression (PCR) or Partial Least Squares (PLS), (Geladi and Kowalski 1986a and b). Among these methods, the PLS method has gained increased support during recent decades (Geladi 1988).

In multivariate calibration the measurement process is modeled by identifying a calibration matrix (or gain matrix) which transforms measured data, $y$, into an estimate of the unknown state vector, $\boldsymbol{x}$. The basis is $\boldsymbol{p}$ samples (or frequently also known as objects) of a representative set of corresponding measurements of both the $n$ -

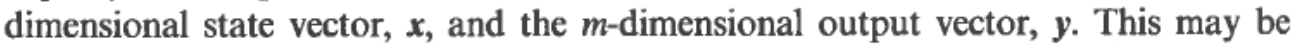
expressed in terms of two matrices $X(p \times n)$ and $Y(p \times m)$ as (Berntsen 1989):

$$
\begin{aligned}
& X=\left[x_{1}-x_{0}, x_{2}-x_{0}, \ldots, x_{p}-x_{0}\right]^{\mathrm{T}} \\
& Y=\left[y_{1}-y_{0}, y_{2}-y_{0}, \ldots, y_{p}-y_{0}\right]^{\mathrm{T}}
\end{aligned}
$$

where $x_{0}$ and $y_{0}$ are the mean values of the $x$ and $y$ vectors:

$$
\begin{aligned}
& x_{0}=\frac{1}{p_{k}} \sum_{k=1}^{p} x_{k} \\
& y_{0}=\frac{1}{p_{k}} \sum_{k=1}^{p} y_{k}
\end{aligned}
$$

In multivariate calibration an estimator $\boldsymbol{h}(\boldsymbol{y})$ is established:

$$
\hat{\boldsymbol{x}}=\boldsymbol{h}(\boldsymbol{y})
$$

where $\hat{\boldsymbol{x}}$ is optimally estimated by an identification method, such as the linear Partial Least Squares (PLS) regression algorithm. The relationship between the measurement vector $\boldsymbol{y}$, and the estimator, $\hat{\boldsymbol{x}}$, of the unknown state vector, $\boldsymbol{x}$, is hence given by:

$$
\hat{\boldsymbol{x}}=x_{0}+K\left(y-y_{0}\right)
$$

The gain matrix $K$ is related to the calibration matrix $B$ used in multivariate calibration as:

$$
K=B^{\mathrm{T}} .
$$

A thorough presentation of multivariate calibration and techniques can be found in the literature (Martens and Næs, 1989).

Analysis has been carried out showing that there is an inner relationship between Multivariate Calibration and the Extended Kalman Filter (EKF), (Berntsen 1989). In addition, a recursive algorithm for PLS regression has recently been presented which will offer a dynamic updating of a calibration model (Helland et al. 1991). Thus it is believed that the integration of MC methods into related dynamic control systems will be further developed in future.

The Partial Least Squares (PLS) algorithm as implemented in the commercially available software package Unscrambler (CAMO 1989) was used throughout the work presented in this paper in order to determine the estimators (Eqn. (5)) and model relations. In multivariate calibration, the nomenclature prediction is frequently used equivalently to estimation when the estimator determines the state vector from new time series that is not part of the calibration (or training) samples. 


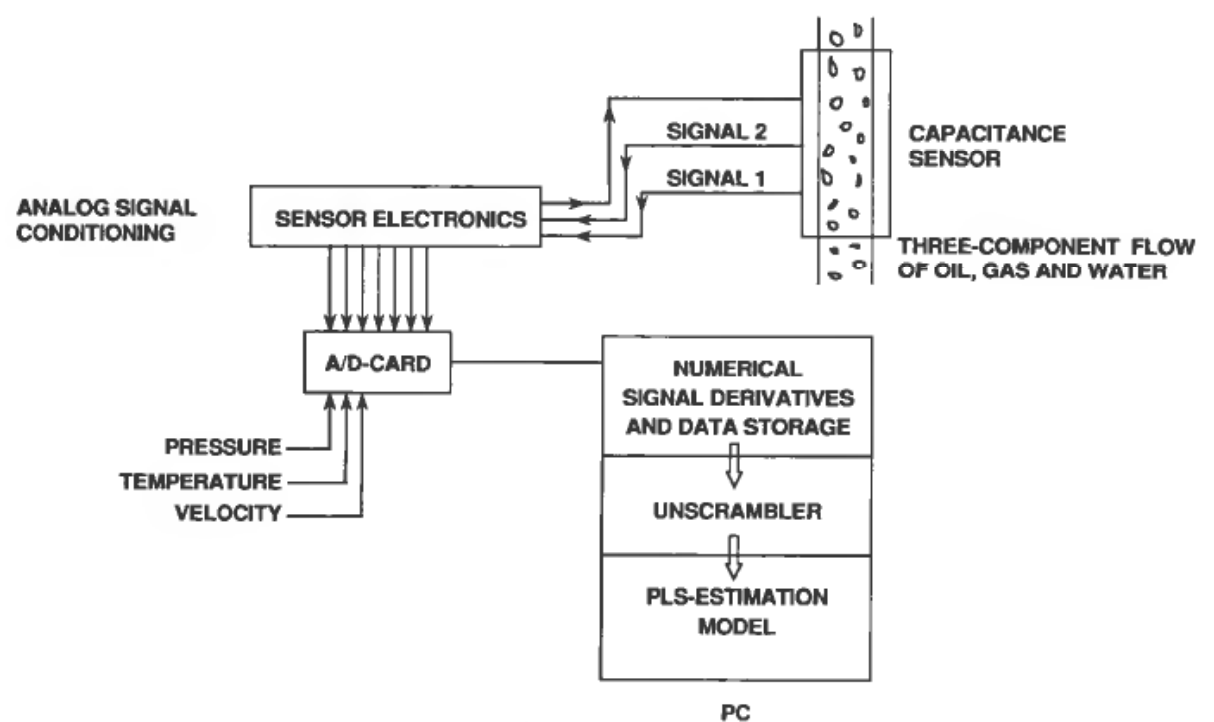

Figure 2. A schematic illustration of the multiphase analysis system.

\section{Signal processing}

A schematic diagram of the measurement system is shown in Fig. 2. The system mainly consists of a capacitance sensor with two detector electrodes and appropriate electronics. The capacitance signals were sampled as discrete time series with 256 sample points at a sample frequency of $200 \mathrm{~Hz}$. These unfiltered primary signals were analysed and signal characteristics extracted.

The capacitance time series were reduced to signal descriptive statistical derivatives in the time and frequency domain. The most important signal derivatives building up the measurement vector, $\boldsymbol{y}$, were found to be:

$d C 1$ the mean value of the capacitance signal $C 1$,

stdC1 the standard deviation of $C 1$,

$f t C 1$ the centre frequency of the power spectrum,

$f w C 1$ the whiteness of the power spectrum,

$d C 1^{2}$ the square term of the capacitance mean value.

Other variables were also derived from the capacitance signals. Some of these were found directly using analogue circuits while others were computed numerically based on the recorded time series. Examples of these are the root of the mean square value of $C 1$ (determined by analogue circuits) and the maximum and minimum values of $C 1$ (computed numerically).

In addition temperature, pressure and a variable related to the velocity of the multiphase flow were used as input variables. The velocity indication was provided by a turbine meter.

\section{Test facility and reference measurements}

The state vector, $\boldsymbol{x}$, contains the multiphase composition variables of gas and water. The gas fraction is conventionally denoted as $\alpha$ and the water fraction as $\beta$. This section presents the test facility and also describes how the reference fraction values of $\alpha$ and $\beta$ 


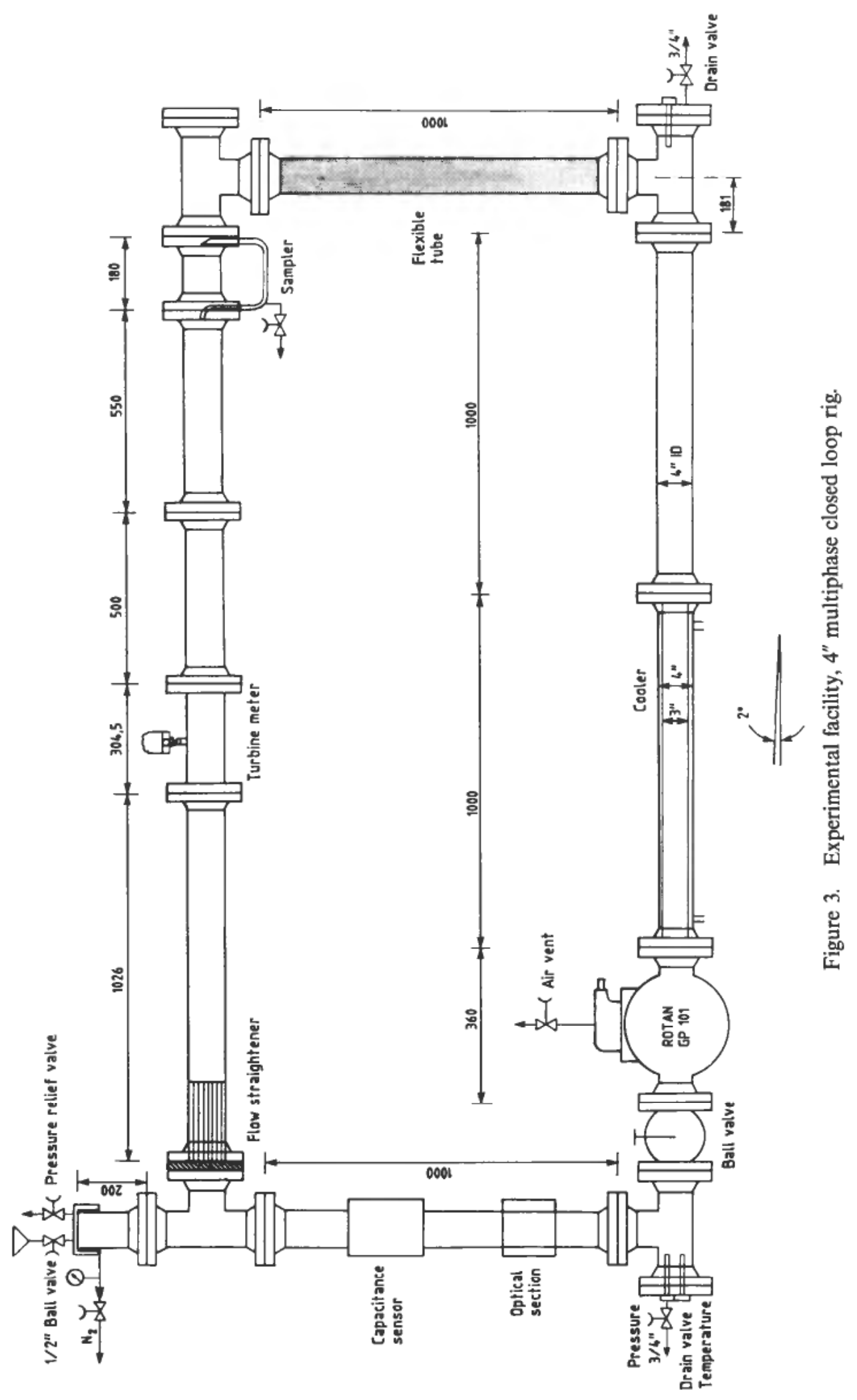


were obtained. The laboratory tests were performed using a $4 "$ multiphase test rig at CMI shown in Fig. 3. The rig is a closed loop and generates bubble gas flow through the vertical test section. As the flow rig is a closed loop configuration with no separation tank, and the gear pump keeps the flow well mixed, the water/oil ratio during a test can be regarded as constant.

The water/oil ratio was determined by liquid sampling and by centrifugal bottom sediment and water (bs\&w) analysis of these samples. In this way the water/oil ratio in the flow was controlled to an uncertainty of better than $\pm 1 \%$. The reference gas fraction is provided by dielectric modeling of the mixture when knowing the watercut (the water/oil ratio), as determined by liquid sampling, is known.

The capacitance sensor was calibrated with fluids of known permittivities so that the bulk permittivity of the mixture is directly represented by the capacitance signal. The permittivity of the oil/water liquid phase was calculated from the measured watercut and known oil permittivity using a resulting permittivity model of a mixture of two dielectric components.

As the bulk permittivity of the liquid phase are measured and the permittivity of the gas is known, the reference gas fraction was determined using Bruggeman's two-component permittivity model (van Beek 1967):

$$
\alpha=1-\frac{1-\varepsilon_{b}}{1-\varepsilon_{s}}\left(\frac{\varepsilon_{s}}{\varepsilon_{b}}\right)^{\frac{1}{3}}
$$

where $\varepsilon_{b}$ is the bulk permittivity, and $\varepsilon_{s}$ is the permittivity of the two-component mixture of oil/water.

To provide an accurate gas fraction reference using the dielectric model (Eqn (8)), the oil/gas/water flow must be well mixed in the test section. In this closed loop configuration of the rig, liquid and gas are circulated to a well-mixed flow until an upper limit is reached where gas hold-up at the top of the rig occurs. It was observed that the maximum gas fraction obtainable in the test section was about $20 \%$. In that gas fraction range, the flow through the test section is restricted to well-mixed bubbly flow where the reference gas fractions are known to an uncertainty of better than $\pm 3 \%$.

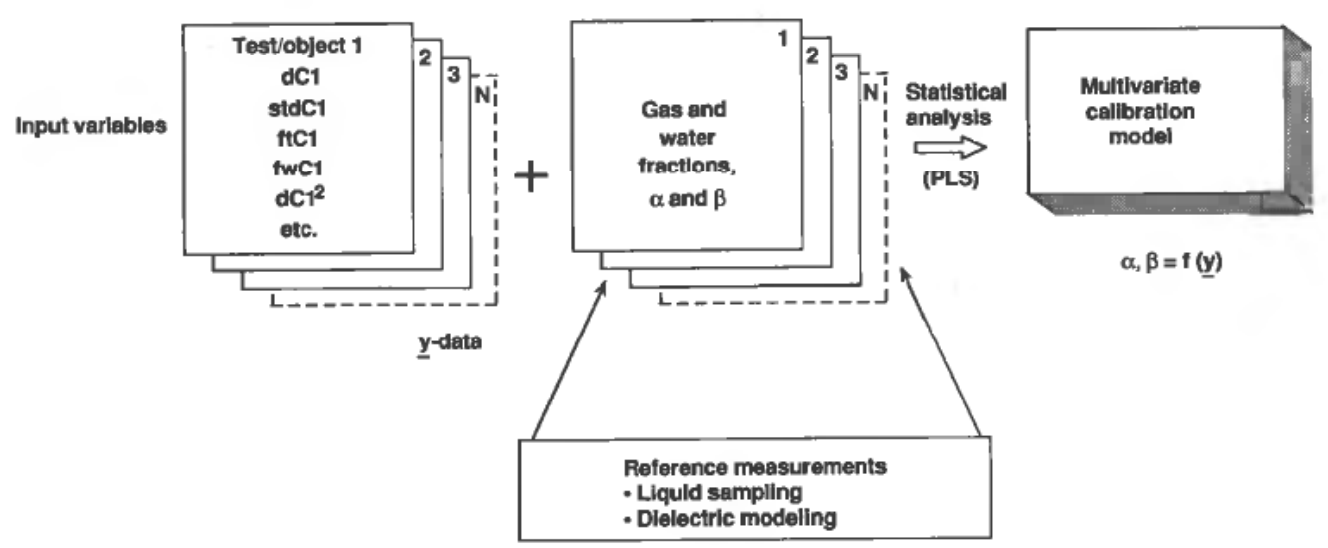

Figure 4. Multivariate modeling of compositions in a multiphase flow. 


\section{Model generation}

The multivariate model was established based on 49 representative calibration samples in which both the state vector, $\boldsymbol{x}$, of flow compositions, and the measurement vector, $\boldsymbol{y}$, of primary variables were known. The model generation is schematically shown in Fig. 4. The calibration tests covered as wide a range of obtainable fractions of gas and water in the rig as possible. The oil was a transformer oil, which when mixed with water, forms an oil continuous liquid phase at water fractions below about $40 \%$. With higher water fractions, the liquid phase becomes water continuous and another sensor principle than capacitance must be utilized.

The following fractions were selected as samples during the calibration tests:

Water/oil ratios: $1,10,20,30,35,40 \%$.

Gas fractions: $\quad 0,7,14,20 \%$.

The on-line capacitance signals, pressure, temperature, and the turbine meter output were all logged along with the off-line determined watercut, by a PC-based data acquisition program. The computer program also calculated the derivatives from the time-varying capacitance signals and the reference gas fraction values. The results of the computations were stored to file and the PLS-modeling was carried out using the Unscrambler.

The PLS-method is a linear estimator. It has been chosen due to its robustness and ability to incorporate many variables. However, a priori it is known that the permittivity of the bulk is non-linearly related to the water fraction. This non-linearity is shown in Fig. 5. The water fraction, $\beta$, is plotted as a function of the mean value of the capacitance signal, $d C 1$, for the 49 calibration objects. It was therefore important to incorporate and use the physical relation in the model that was already known. For this reason the square of the mean capacitance was included due to the prior knowledge of this non-linearity in the data. Consequently knowledge of the process which is modeled, is very important and must be viewed in the light of the estimation method used. The mutual relations between the most important input variables and the fractions to be estimated by the model, are visually illustrated in Fig. 6. The square of

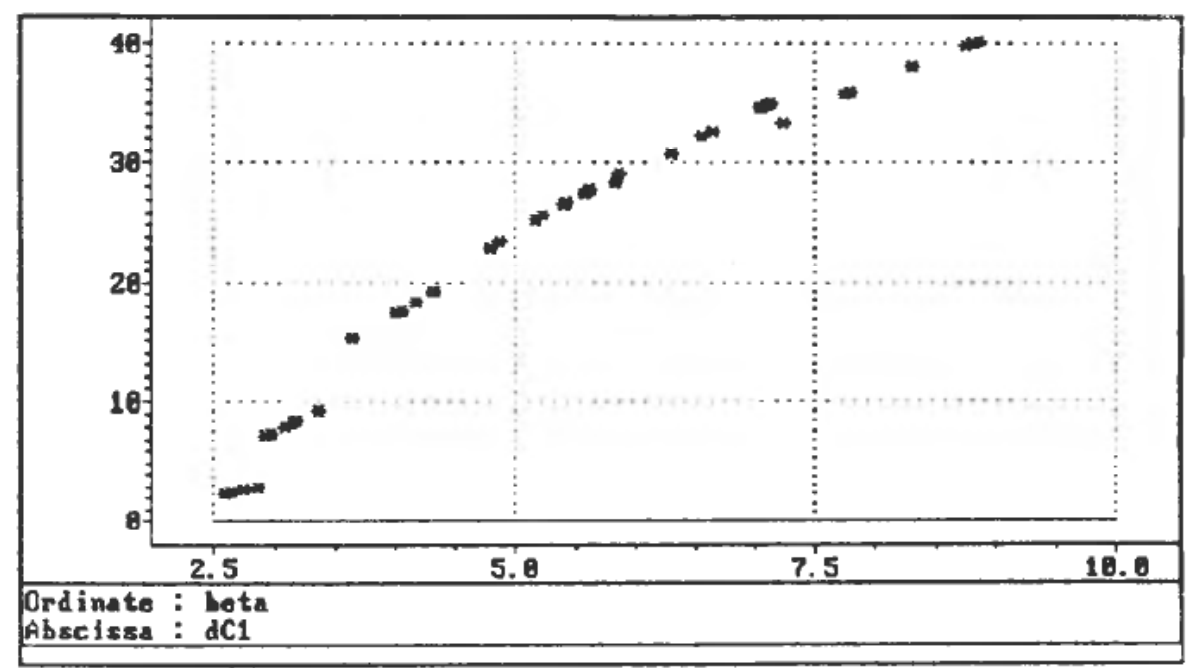

Figure 5. The water fraction as a function of the mean value of the capacitance signal. 


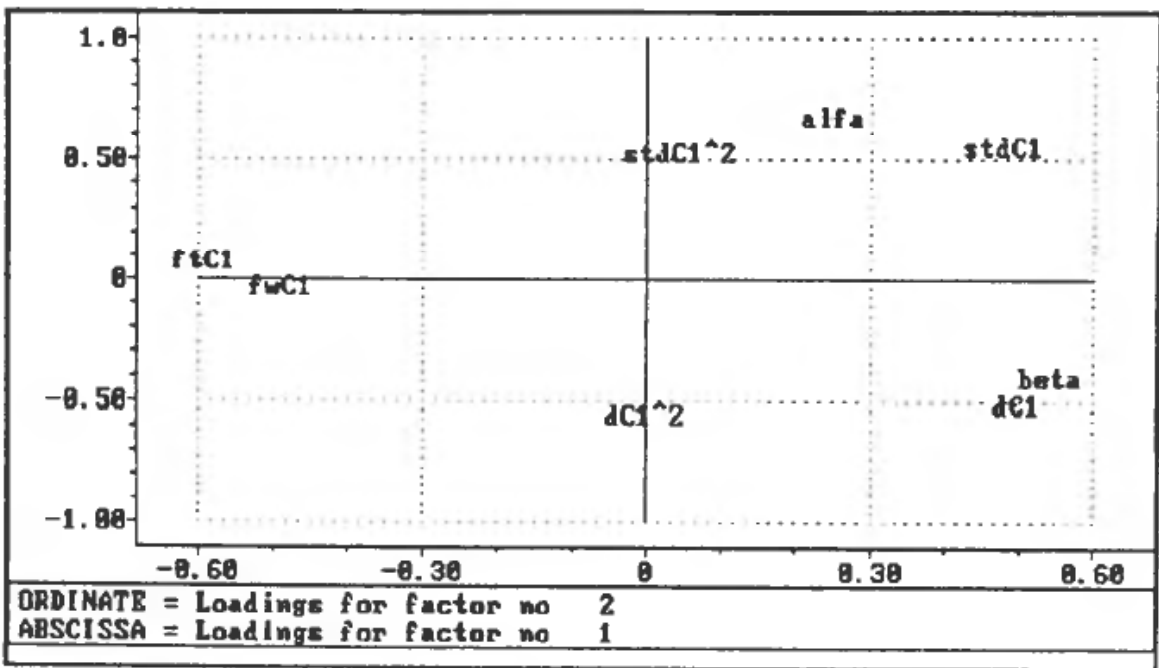

Figure 6. A visual illustration of the mutual relations between the most important variables used in the model.

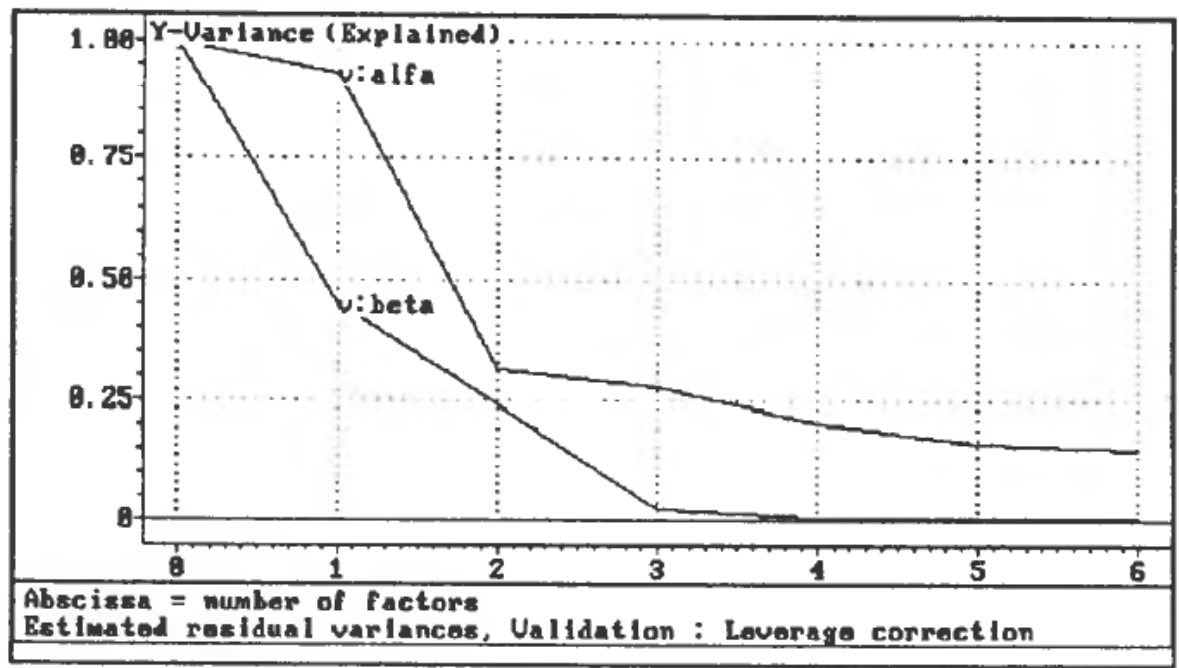

Figure 7. Explained variance in the validation set during model generation.

the standard deviation of $C 1, s t d C 1$, has also been included. Briefly, it is qualitatively observed that the water fraction is mostly modeled by the mean capacitance level while the variance of the capacitance signals provides significant information about the gas content. The different locations of the square variables relative to their origins show that non-linear modeling improves the estimations. This is confirmed by experimental investigations.

This paper is not intended to give a detailed description of the PLS-modeling. However, it should be noted that the plot-axes of Fig. 6 are principal components frequently also denoted as factors or latent variables. These are estimated linear combinations of the original measurements and may be viewed as hidden summarizing 
variables that are not directly measurable. In the multivariate analysis the number of variables is reduced to fewer factors which explain most of the variance in the data structure considered (Anderson 1958). An example is given in Fig. 7.

It should also be mentioned that the user of these techniques, must be aware that artificial correlations to the model are potentially included, and that these have the experimental set up and not physical reality as their origin. A typical example of such a relation from this work might be a correlation between the temperature and gas fraction due to the temperature increase during the experimental tests, and that the calibration experiments started with the lowest gas fraction and was then increased. As a result, the data set produced a false proportional relation between the temperature and gas fraction. This false relationship was removed by the suppression of the variable during the modeling using appropriate weighting. However, this may be avoided using a more suitable experimental design when planning and carrying out the calibration experiments.

The linear PLS-method has been used in this work. If required, this multivariate analysis could equally well have been carried out using a non-linear estimator (see Eqn (5) above). Artificial neural networks may beneficially offer such non-linear transforms (Qin and McAvoy 1991). The iterative numerical PLS-algorithm is then replaced by a multilayer net architecture model which simulates a biological neural network (Widrow and Lehr 1990).

\section{Results}

The multivariate model was validated using 12 measurement series that were not part of the calibration set of 49 objects. This evaluation corresponded to on-line computations of gas and water fractions. In addition, reference gas and water fraction values were found off-line. Figs 8 and 9 show model predictions of the gas and water fractions. The absolute deviations found between estimated fractions and corresponding reference values are within $\pm 5 \%$ for gas (range $0-20 \%$ ) and within $\pm 2 \%$ for water (range $0-40 \%$ ).

One major advantage of this technique is that the modeling of the flowing medium is to a large extent taken care of by the multivariate calibration procedure. A disadvantage is that a model calibration made in the laboratory to some degree will be installation dependent and may not be suitable for an actual installation. The accuracy and representativity of the reference measurements will therefore affect the overall accuracy of the final prediction. An in situ calibration against a reference system may be necessary to achieve sufficiently accurate measurements in an industrial application. However, this will depend on the accuracy required and as this has only been a feasibility study, further work is obviously necessary to make an exact quantification of figures.

\section{Further work}

For a capacitance sensor the bulk capacitance $\left(C_{b}\right)$ is proportional to the permittivity of the flow as:

$$
C_{b}=C_{0} \varepsilon_{b}
$$

where $C_{0}$ is the sensor constant (the bulk capacitance with empty sensor), and $\varepsilon_{b}$ is the permittivity of the bulk (relative to vacuum). 


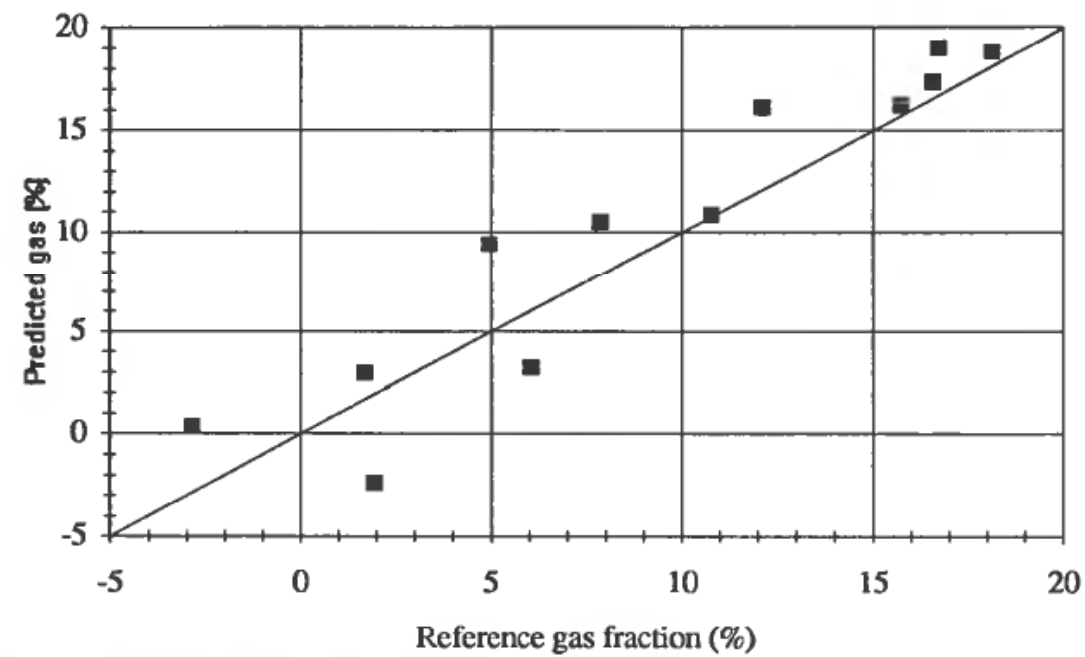

Figure 8. Gas fractions computed by multivariate modeling compared to independent reference data.

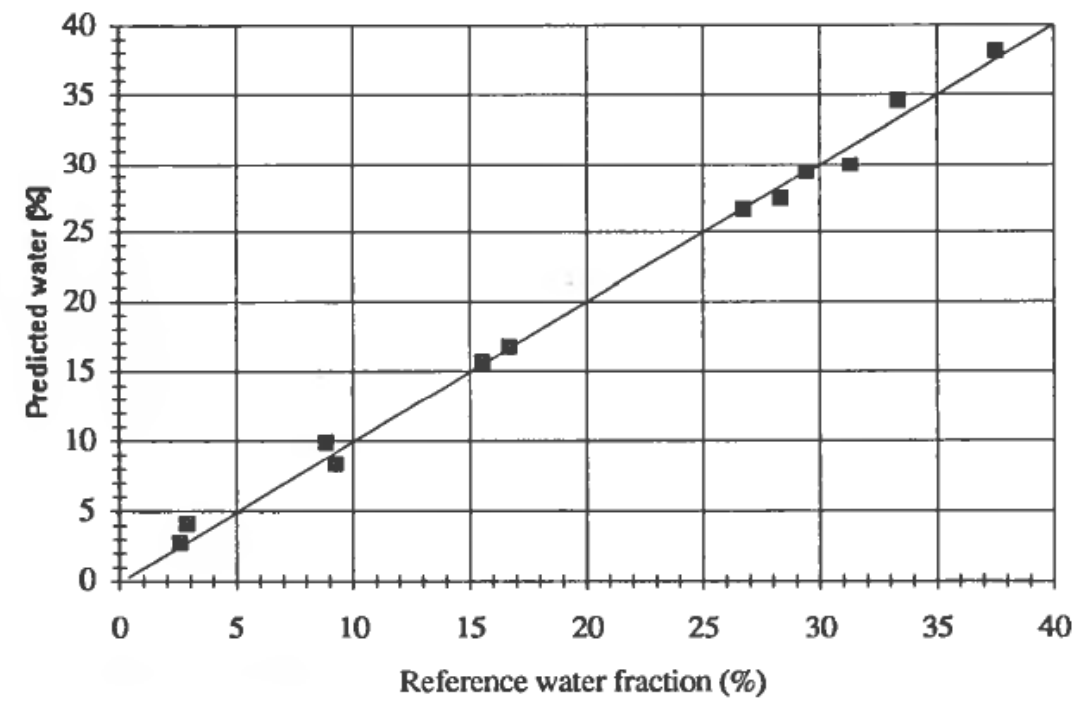

Figure 9. Water fractions computed by multivariate modeling compared to independent reference data.

The sensor constant, $C_{0}$, is dependent on the design and size of the sensor. The multivariate model in this work has been computed from measurement series where a sensor with a certain, $C_{0}$, has been used. This model will not be suitable for another sensor with a diverging sensor constant. Changes of the sensor electronics will equivalently cause the model to fail and a new demanding in situ multivariate calibration may be necessary. This corresponds to empirical black box modeling of the total system of the sensor, electronics and partial unknown physical relations in the flow. This one level modeling may be sufficient for some applications. However, flexibility is important in industrial measurement systems; i.e. it is advantageous if transducers or electronics can be substituted, while still keeping the models and accuracy intact. 

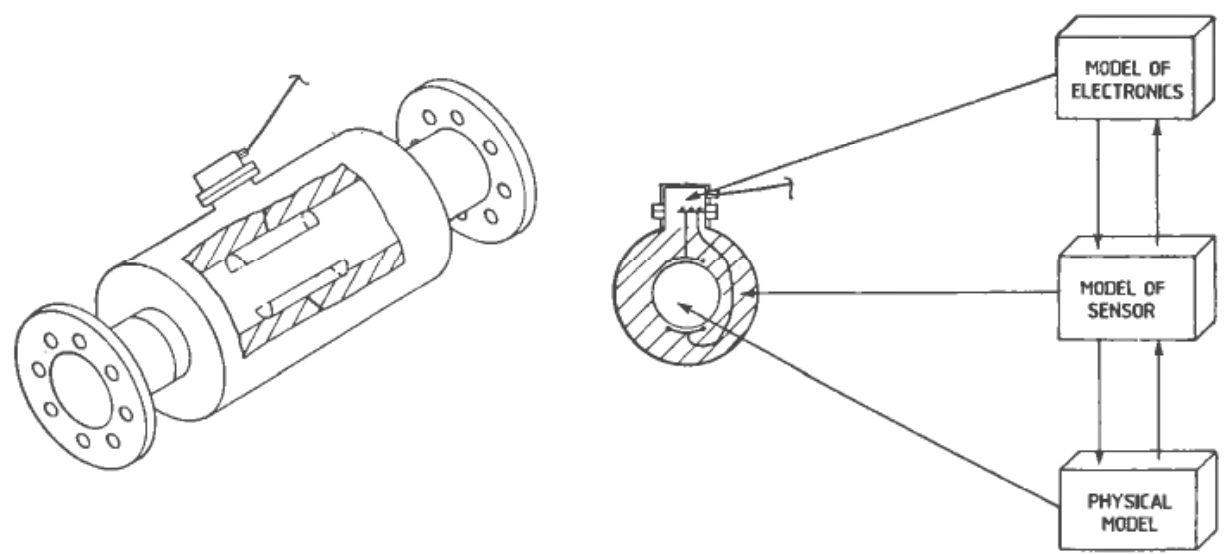

Figure 10. Multi-level modeling of an instrumentation system.

This can be done by static and separate calibration of sensor and electronics. Two relations, a permittivity-capacitance relation for the sensor and a capacitance-voltage relation for the electronics are then found. In this way the digitized voltage values can be converted by software to permittivity time series where signal processing can be applied and an estimation model found. Such conversions to physical quantities should also be applied to other variables, e.g. temperature, pressure, density, etc. In this way a more universally valid and physically true model is obtained with permittivity (and temperature and pressure) and not voltage signals as a basis. The flow estimation model may then be used in combination with different sensors (of the same type). If one wants to use the sensor for an entirely different flow, the flow model can be changed while keeping the models of the rest of the system unmodified. A schematic description of such a capacitance measurement system is given in Fig. 10.

\section{Conclusions}

The results of this feasibility study show that the integrated use of signal processing and multivariate calibration may be used in applications where advanced measurement techniques and control systems are required.

Time series of capacitance signals have been characterized by statistical derivatives in the time and frequency domain. The experimental results in Figs 8 and 9 verify that it is possible to estimate fractions of oil, water and gas in multiphase flow using only a single capacitance sensor.

If a fast, dedicated computer is employed, the estimation can be done on-line. The measuring time is mainly dominated by the duration of the data-logging. The numerical data processing capacity can be adapted so that all computations can be carried out within the time span of the data acquisition. The time response for the system will then only be limited by the physical process, i.e. the time needed to gather the time series. This will usually be low, within $2-4$ seconds, depending on the number of sample points required and the sampling frequency the time series are recorded.

Using further signal processing of the signals from a multiple-electrode capacitance sensor, the composition measurements may be complemented by velocity measurements to provide the individual flow rates of oil, gas and water. Depending on the accuracy required, one may then see the possibility of developing a non-intrusive flow 
rate meter without using any gamma densitometer. One application of this concept is monitoring three-phase flow from deep water satellites.

Other applications in the process industry may also be identified. Monitoring of flow regimes during multiphase transport of oil/water/gas mixtures and flow patterns during pneumatic conveying of solids are advantageous for accurate mass flow rate measurements.

The multivariate calibration method is to a large extent an empirical modeling of hidden physical relations that frequently cannot be thoroughly described by known mathematical representations. However, pre-treatment of the signals to provide universal physical variables as input to the model is strongly recommended. Further, it is important to incorporate a priori knowledge of the process, e.g. non-linearities, physical relations and known calibration data, thereby making the model even more robust and generally applicable.

This multivariate approach is, in principle, a general technique and may also be used by other types of sensors than capacitance or, if desired, applied to quite other processes or problem situations where complicated measurement problems occur.

\section{REFERENCES}

ANDERSON, T. W. (1958). An Introduction to Multivariate Statistical Analysis (Wiley), pp. 272 287.

BERNTSEN, H. (1989). Multivariate calibration as an identification method and its relationship to the extended Kalmanfilter. Proceedings of the 28th Conference on Decision and Control, Tampa, Florida. December 1989. Vol. 1 (IEEE, New York), pp. 640-645.

CAMO (Computer Aided Modelling) A/S, UNSCRAMBLER User's guide, Trondheim, Norway (1989).

Dykesteen, E. and Frantzen, K. H. (1990). The CMI multiphase fraction meter. IBC Technical Services Ltd. The International Conference on Basic Principles \& Industrial Applications of Multiphase Flow, London, 24-25 April 1990.

GELADI, P. (1988). Notes on the history and nature of partial least squares (PLS) modelling. Journal of Chemometrics, 2, 231-246.

GELADI, P. and KowALSKI, B. R. (1986a). Partial least squares regression: a tutorial. Analytica Chimica Acta, 185, 1-17.

GELADI, P. and KoWALSKI, B. R. (1986b). An example of 2-block predictive partial least squares regression with simulated data. Analytica Chimica Acta, 185, 19-32.

HAMmER, E. A. (1983). Three-component flow measurement in oil/gas/water mixtures using capacitance transducers. Ph.D. Thesis.

Helland, K., Berntsen, H. E., Borgen, O. S. and Martens, H. (1992). Recursive algorithm for partial least squares regression. Chemometrics and Intelligent Laboratory Systems, 14, 129137 (Elsevier Science Publishers B.V., Amsterdam).

Martens, H. and NÆS, T. (1989). Multivariate Calibration (Wiley).

QıN, S., and McAvor, T. J. (1991). Annual meeting of the American Institute of Chemical Engineers, Los Angeles, California, 17-22 November 1991. Paper 149d. (AIChE, New York).

TAITEL, Y., BORNEA, D. and DuKLER, A. E. (1980) Modelling flow pattern transitions for steady upward gas-liquid flow in vertical tubes. AICHe Journal, 26, 345-354.

VAN BEEK, L. (1967). Dielectric behaviour of heterogeneous systems. Progress in Dielectrics, 7, 71113.

Widrow, B. and LeHR, M. A. (1990). 30 Years of adaptive neural networks: Perceptron, Madaline, and backpropagation. Proceedings of the IEEE, 78, 1415-1442. 